Physics Vol. 2, No. 2, pp. 71-79, 1965. Physics Publishing Co. Printed in Great Britain.

\title{
ВЛИЯНИЕ ПРИМЕСНОГО ФЕРРОМАГНЕТИЗМА И ВНЕШНЕГО МАГНИТНОГО ПОЛЯ НА СОПРОТИВЛЕНИЕ МЕТАЛЛА С МАГНИТНЫМИ ПРИМЕСЯМИ
}

\section{A.A. АБРИКОСОВ}

(Получили: 1 апреля 1965)

Расчет, произведенный в [1] распространен на случай примесного ферромагнетизма и внешнего магнитного поля. Показано, что в зависимости от соотношения между $T_{\max }(\mathrm{cm} \cdot[1]), T_{c}$ (температуры Кюри) и $\mu H$ возможны различные виды немонотонной зависимости сопротивления от температуры. Оказывается также, что сопротивление может уменьшаться с маГнитным полем.

В предыдущей работе [1] было рассмотрено электрическое сопротивление немагнитного металла с малой примесью магнитных атомов. При этом оказалось, что часть сопротивления, связанная с обменным взаимодействием электронов с примесными атомами меняется с температурой. Если это взаимодействие имеет антиферромагнитный знак $(J<0)$, то эта часть сопротивления растет при понижении температуры и при некоторой температуре имеет максимум, после чего начинает падать. Температура не зависит от концентрации примеси.

На опыте (см. например [2]) были получены кривые с максимумом, однако, положение максимума существенно зависело от концентрации примеси. В настоящей работе показано, что это получается в результате примесного ферромагнетизма. Рассмотрено влияние на сопротивление внешнего магнитного поля $(*)$

\section{1. Примесный ферромагнетизм}

для учета примесного ферромагнетизма и действия внешнего магнитного поля мы поступим в духе работы [3]. Из диаграмм для $G$ и $\mathscr{G}$ функций (см. [1]) мы выделим диаграммы первого порядка, изображенные на рис.1. Эти диаграммы равны нулю при отсутствии упорядочения спинов и отличны от нуля в рассматриваемом случае. Их можно рассматривать, как результат действия некоторого эффективного «поля》 в одном случае на спин электрона, а в другом случае на спин примеси. Мы объединим это «поле» с настоящим магнитным полем и будем рассматривать примесь и электрон под действием некоторого суммарного поля, которое мы затем определим самосогласованным методом. «Свободные» гриновские функции будут иметь вид:

$$
G=\frac{1}{i \omega-\xi+\sigma_{z} P} \quad \text { (a) } \quad \mathscr{G}=\frac{1}{i \omega-\lambda+S_{Z} Q}
$$

*) Влияние внешнего магнитного поля на сопротивление металлов с магнитными примесями было рассмотрено другим способом Л.Гуревичем и Яссиевичем. Эффект Кондо не учитывался. 
Обозначения те же, что и в [1].

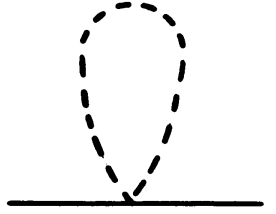

(a)

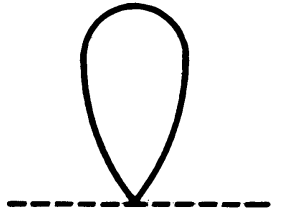

(ठ)

Рис. 1

Заметим, что процесс усреднения по спинам примеси в данном случа должен производиться с учетом «поля》 $Q$. Ввиду этого нормирующий множитель равен не $e^{\lambda / T /(2 S+1)}$, а

$$
e^{\lambda / T} /\left(\sum_{-s}^{s} e^{-M Q}\right)=e^{\lambda / T} \frac{\sinh (Q / 2 T)}{\sinh [Q(S+1 / 2) / T]}
$$

Из диаграмм la и $\mathrm{b}$, и формул (1) получаем самосогласованные урав нения для $P$ и $Q$ (здесь и дальше $\hbar=1$ ):

$$
\begin{aligned}
& Q=g \mu_{0} H+\frac{J}{N} \frac{p_{0} m}{2 \pi^{2}} S p_{\sigma} T \sum_{\omega} \int d \xi \frac{\sigma_{Z}}{i \omega-\xi+\sigma_{Z} P} \\
& P=\mu_{0} H+\frac{N_{i} J}{N} \frac{e^{\lambda / T} \sinh (Q / 2 T)}{\sinh [Q(S+1 / 2) / T]} S_{p s} T \sum_{\omega} \frac{S_{Z}}{i \omega-\lambda+S_{Z} Q}
\end{aligned}
$$

$\left(N_{1}\right.$ - число атомов примеси в $l \mathrm{~cm}^{3}, \mu_{\circ}$ - магнетон Бора, $g \approx 2$ гиромагнитный фактор примеси). Взяв суммы и интегралы и считая $\lambda \gg T$, получаем:

$$
\begin{aligned}
& P=\mu_{0} H+J C S B S\left(\frac{S Q}{T}\right) \\
& Q=g \mu_{0} H+\frac{3 Z J}{2 \varepsilon_{F}} P
\end{aligned}
$$

$\left(B_{s}(x)=\frac{2 S+1}{2 S} \operatorname{coth} \frac{(2 S+1) x}{2 S}-\frac{1}{2 S} \operatorname{coth} \frac{x}{2 S}\right.$ - Функция Бриллюэна, $z$ - число электронов на 1 атом, $c$ - атомная концентрация). Если подставить $2 \mathrm{~b}$ в 2a, то мы получим в точности условие (13) работы [3] (обозначения $\left.S=\frac{\chi_{0}}{2 \mu_{0}{ }^{2}} P, \frac{\chi_{0}}{\mu_{0}{ }^{2}}=\frac{p_{0} m}{\pi^{2}}, a=\frac{2 J}{N}\right)$. С помощью уравнений (2) можно найти $P$ и $Q$, а следовательно и «свободные» гриновские функции . Для дальнейщего нам понадобятся некоторые оценки и предельные 
выражения. Пусть $\mathrm{H}=0$. Считая аргумент в $B_{\boldsymbol{s}}$ малым и разлагая по нему в ряд, получаем соотношение:

$$
P^{2}=\frac{10 C S(S+1) \varepsilon_{F}}{3 Z\left(S^{2}+S+1 / 2\right)}\left(T_{C}-T\right)
$$

где $T_{\boldsymbol{c}}$ - температура Кюри, равная

$$
T_{C}=\frac{J^{2} C Z S(S+1)}{2 \varepsilon_{F}}
$$

Формула (3) справедлива вблизи $T_{c}$. Если же $T \ll T_{c}$, то

$$
P=J C S
$$

Что касается величины $Q$, то она, согласно (2), равна

$$
\begin{gathered}
Q=J\left[\frac{15}{2} \frac{C S(S+1) Z}{\varepsilon_{F}\left(S^{2}+S+1 / 2\right)}\left(T_{C}-T\right)\right]^{1 / 2}, \quad T_{C}-T \ll T_{C} \\
Q=\frac{3 C Z J^{2} S}{2 \varepsilon_{F}}, \quad T \ll T_{C}
\end{gathered}
$$

Таким образом, при $T_{\boldsymbol{c}}-T \ll T_{\boldsymbol{c}}-Q S \ll T_{\boldsymbol{c}}$, а при $T \lesssim T_{\boldsymbol{c}}-Q S \sim T_{\boldsymbol{c}}$.

Теперь рассмотрим, что происходит при наличии поля. Нас будет интересовать случай $\mu_{0} H \gg T_{c}$ и $T \gg T_{\boldsymbol{c}} \cdot$ Считая опять аргумент $B_{s}$ малым, получаем:

$$
P=\mu_{0} H\left(1+\frac{J C S(S+1) g}{3 T}\right)
$$

Условием применимости этой формулы является $g \mu_{0} S H / T \ll 1$. В обратном предельном случае

$$
P=\mu_{0} H+J C S
$$

Из (2) находим, что в обоих предельных случаях

$$
Q \approx g \mu_{0} H
$$

Эти формулы будут полезны нам в дальнейшем •

\section{2. Собственно энергетическая часть}

Теперь рассмотрим рассеяние. В данном случае имеется несколько типов диаграмм, дающих вклад в собственно-энергетическую часть. Одна диаграмма соответствует [1] (рис.2). Остальные изображены на рис. 3, где оба взаимодействия в каждой диаграмме относятся к одному атому (для простоты мы ограничились борновским приближением для обычного рассеяния). Диаграммы на рис. 3 равны нулю при отсутствии 
поляризации спинов (*). Их вклад вычисляется очень просто и дает (во временно́й гриновской функции)

$$
\sum_{\alpha \alpha^{\prime}}^{(1)}=-i \pi \operatorname{sign} \omega \frac{p_{0} m}{2 \pi^{2}}\left[-2 f_{0}\left(P-\mu_{0} H\right) \sigma_{Z}+\frac{1}{N_{i}}\left(P-\mu_{0} H\right)^{2}\right]_{\alpha \alpha^{\prime}}
$$

где $f_{0}$ - амплитуда обычного рассеяния (предполагаемого для простот изотропным), а $P$ определено в предыдущем разделе. Что касается

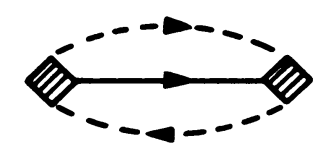

Рис. 2

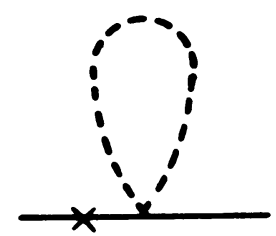

(a)

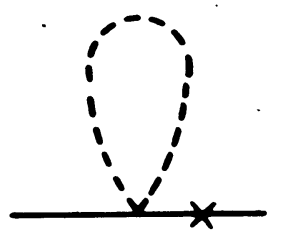

(ठ)

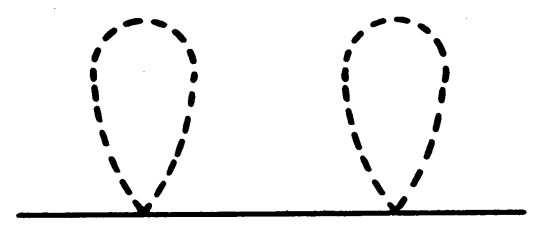

(b)

Рис. 3

диаграммы на рис. 2, то она вычисляется аналогично тому, как это было сделано в [1]:

$$
\begin{aligned}
& \sum_{\alpha \alpha^{\prime}}^{(2)}=i \operatorname{sign} \omega \frac{e^{\lambda / T_{\sinh }(Q / 2 T)}}{\sinh [(S+1 / 2) Q / T]} N_{i} \frac{p_{0} m}{2 \pi^{2}} \\
& x \int \Gamma_{\alpha \beta}, \alpha_{1} \beta_{1}\left(\omega, \omega_{1} ; \omega+\omega_{1}-\omega_{2}, \omega_{2}\right) \Gamma_{\alpha_{2} \beta_{2}, \alpha^{\prime} \beta_{3}}\left(\omega+\omega_{1}-\omega_{2}, \omega ; \omega_{1} \omega_{1}\right)
\end{aligned}
$$

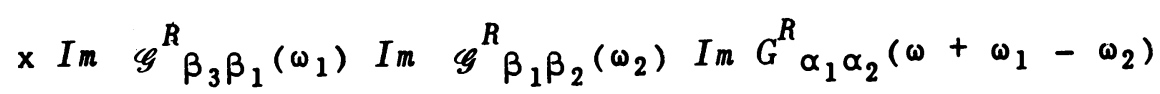

$$
\begin{aligned}
& \times\left(\tanh \frac{\omega_{2}}{2 T}-\operatorname{coth} \frac{\omega_{2}-\omega}{2 T}\right)\left(\tanh \frac{\omega_{1}}{2 T}-\tanh \frac{\omega+\omega_{1}-\omega_{2}}{2 T}\right) \frac{d \omega_{1}}{2 \pi} \frac{d \omega_{2}}{2 \pi}
\end{aligned}
$$

*) В письме в редакцию ЖЭТФ, где очень кратко изложены результаты настоящей работы, эти члены не были учтены. Автор благодарен А.Русинову, обратившему на них его внимание. 
Подставляя

$$
\begin{aligned}
& \text { Im } \mathscr{G}_{\beta \beta_{1}}^{R}=-\pi \delta_{\beta \beta_{1}}\left(\omega-\lambda+S_{Z \beta} Q\right) \\
& \text { Im } G_{\alpha \alpha_{1}}^{R}=-\pi \delta_{\alpha \alpha_{1}}\left(\omega-\xi+\delta_{Z \alpha} P\right)
\end{aligned}
$$

взяв интегралы по $\omega_{1}, \omega_{2}$ и $\xi$, и учитывая, что $\lambda \gg T$, получаем

$$
\begin{aligned}
& \sum_{\alpha \alpha^{\prime}}^{(2)}=-i \operatorname{sign} \omega N_{i} \frac{p_{0} m}{2 \pi} \frac{\sinh (Q / 2 T)}{\sinh [Q(2 S+1) / 2 T]} \frac{e^{\omega / T+1}}{e^{\omega-Q S_{Z \beta} / T}-e^{-Q S_{Z \beta_{1}} / T}} \\
& \times \Gamma_{\alpha \beta, \alpha_{1} \beta_{1}}\left[\omega, \lambda-Q S_{Z \beta^{\prime}} ; \omega+Q\left(S_{Z \beta_{1}}-S_{Z \beta}\right), \lambda-Q S_{Z \beta_{1}}\right] \\
& \times \Gamma_{\alpha_{1} \beta_{1}, \alpha^{\prime} \beta}\left[\omega+Q\left(S_{Z \beta_{1}}-S_{Z \beta}\right), \lambda-Q S_{Z \beta_{1}} ; \omega, \lambda-Q S_{Z \beta}\right]
\end{aligned}
$$

Ограничиваясь логарифмической точностью, мы можем, как и в [1], вычислять $\Gamma$ с помощью «временно́й» техники при $T=0$, и положить везде $\lambda=0$. В [1] нижним пределом логарифмического интеграла была величина $\omega$, и при вычислении проводимости оказывались существенными $\omega \sim T$. В данном случае положение меняется. Рассмотрим простейщую диаграмму на рис. 4.

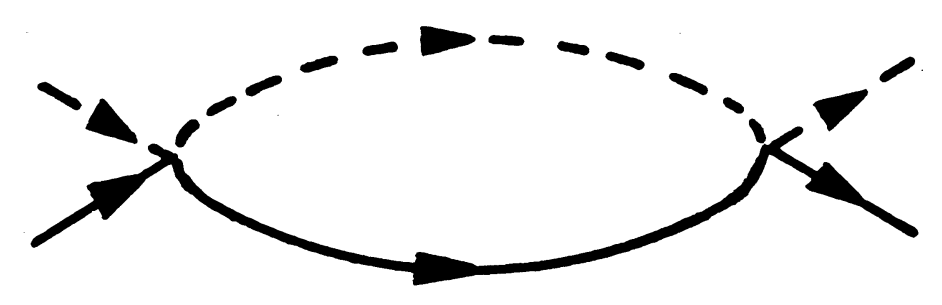

Рис. 4

Аналогично [1] (формула (10а)) получаем:

$$
\begin{aligned}
& -i\left(\frac{J}{N}\right)^{2}(\vec{\sigma} \vec{S})_{\alpha \beta, \alpha_{1} \beta_{1}}(\vec{\sigma} \vec{S})_{\alpha_{1} \beta_{1}, \alpha^{\prime} \beta^{\prime}} \frac{p_{0} m}{2 \pi^{2}} \int \frac{d \omega_{1}}{2 \pi} \int d \xi_{1} \\
& \times \frac{1}{\omega_{1}+Q S_{Z \beta_{1}}+i \delta} \frac{1}{\omega-\omega_{1}-\xi_{1}+P \sigma_{Z \alpha_{1}}+i \delta \operatorname{sign}\left(\xi_{1}-P \sigma_{Z \alpha_{1}}\right)} \\
& =\left(\frac{J}{N}\right)^{2}(\vec{\sigma} \vec{S})_{\alpha \beta, \alpha_{1} \beta_{1}}(\vec{\sigma} \vec{S})_{\alpha_{1} \beta_{1}, \alpha^{\prime} \beta^{\prime}} \frac{p_{0} m}{2 \pi^{2}} \int_{0}^{\varepsilon_{F}} \frac{d \xi_{1}}{\xi_{1}-Q S_{Z \beta_{1}}-\omega-i \delta}
\end{aligned}
$$


(здесь сделана замена $\left.\xi_{1}-P \sigma_{z a} \rightarrow \xi_{1}\right)$. Отсюда видно, что величина $P$ вообще не участвует в логарифмическом интеграле. Нижним предело интеграла оказывается наибольшая из величин $Q$ и $\omega$, т.е. в окончательном результате $\max (Q, T)$. Так как логарифмический интеграл идет по области $\xi_{1} \gg \max (Q, T)$, то выражение (1I) сохраняет ту же спинорную форму, что и при отсутствии упорядочения спинов. Это же относится ко всем следующим порядкам для $\Gamma$.

Таким образом, в выражении (10) в качестве $\Gamma_{\alpha \beta}, \alpha_{1} \beta_{1} \Gamma_{\alpha_{1} \beta_{1}, \alpha^{\prime} \beta}$ можно, согласно [1], подставить

$$
\begin{aligned}
& \left(\begin{array}{ll}
\vec{\sigma} & \vec{S}
\end{array}\right)_{\alpha \beta_{1}, \alpha_{1} \beta_{1}}(\vec{\sigma} \vec{S})_{\alpha_{1} \beta_{1}, \alpha^{\prime} \beta} \\
& {\left[1+\frac{3 J Z}{2 \varepsilon_{F}} \ln \frac{\varepsilon_{F}}{\max (Q,|\omega|)}\right]^{2}}
\end{aligned}
$$

Считая $|\omega| \sim T$, мы получаем, согласно предыдущему разделу, что если магнитное поле отсутсвует или $g \mu_{\circ} H S \ll T_{C}$, то при $T>T_{c}$ в (12) стоит $\ln \frac{\epsilon_{F}}{T}$, а при $T \lesssim \epsilon_{c} \ln \frac{\epsilon_{F}}{T_{c}} \cdot$ Если же $g \mu_{0} H S \gg T_{c}$, то при $т \gg g \mu_{0} H S$ имеем $\ln \frac{\epsilon_{F}}{T}$, а при $T \ll g \mu_{0} H S \quad \ln \frac{\epsilon_{F}}{g \mu_{0} H S}$.

Однако в данном случае зависимость от температуры происходит не только от $Г$, но и от предыдущего множителя в (10). Пользуясь спинорной формой $\Gamma,(12)$ мы получаем

$$
\begin{aligned}
& \sum_{\alpha \alpha^{\prime}}^{(2)}=-i \operatorname{sign} \omega N_{i} \frac{p_{0} m}{2 \pi} \frac{\sinh (Q / 2 T)}{\sinh [Q(2 S+1) / 2 T]} \frac{1}{\left[1+\frac{3 J Z}{2 \varepsilon_{F}} \ln \frac{\varepsilon_{F}}{\max (Q,|\omega|)}\right]^{2}} \\
& \quad \times \sum_{M=-S}^{S} e^{Q M / T}\left[M^{2}+\frac{1}{2}(S+M)(S-M+1)\left(1-\sigma_{Z}\right) \alpha \alpha^{\circ} \frac{e^{\omega / T}}{e^{\omega / T}+e^{Q / T}}\right. \\
&\left.+\frac{1}{2}(S+M+1)(S-M)\left(1+\sigma_{Z}\right) \alpha \alpha^{\prime} \frac{e^{\omega / T}}{e^{\omega / T}+e^{-Q / T}}\right]
\end{aligned}
$$

Взяв сумму по $M$, находим:

$$
\begin{gathered}
\sum_{ \pm}^{(2)}=-i \operatorname{sign} \omega N_{i} \frac{p_{0} m}{2 \pi} \frac{1}{\left[1+\frac{3 J Z}{2 \varepsilon_{F}} \ln \frac{\varepsilon_{F}}{\max (Q,|\omega|)}\right]^{2}} \\
\times\left\{S(S+1)+S B_{S}\left(\frac{S Q}{T}\right)\left[\frac{e^{\omega / T}+1}{e^{\omega / T}+e^{\mp Q / T}}\left(\operatorname{coth} \frac{Q}{2 T} \mp 1\right)-\operatorname{coth} \frac{Q}{2 T}\right]\right\}
\end{gathered}
$$

где знаки + и - относятся к разным ориентациям электронного 
спина. Множитель в $\Sigma^{(1)}+\Sigma^{(2)}$ при $-i \operatorname{sign\omega }$ мы обозначим через $1 / 2 \tau_{ \pm}$. Необменное рассеяние дает, как и раньше (см. [1]) дополнительное слагаемое в $\Sigma$, значительно больщей величины (кроме окрестности «езонанса») .

Аналогично [1] получаем для проводимости

$$
\sigma=\frac{Z N e^{2}}{2 m} \int_{0}^{\infty} \frac{d \omega}{2 T \cosh \frac{2 \omega}{2 T}}\left[\tau_{+}{ }^{\prime}(\omega)+\tau_{-}^{\prime}(\omega)\right]
$$

здесь $\tau_{t}{ }^{\prime}(\omega)=\left[1 / \tau_{\text {ord }}+1 / \tau_{+}(\omega)\right]^{-1}$. Мы воспользовались тем, что величина $\tau_{+}^{\prime}(\omega)+\tau_{-}^{\prime}(\omega)$ симметрична относительно изменения знака $\omega$. Считая $1 / \tau_{\text {n }} \gg 1 / \tau_{+}(\omega)$ получаем $\rho=\rho$ ord $+\rho$ ex (в $\rho$ ex включена и интерференционная часть; соответствующий член в $\Sigma^{(1)}$ дх дает вклад во втором порядке по $\mathrm{J}\left(\mathrm{f}_{0}\right)$,

$$
\begin{gathered}
\rho_{\text {ex }}=\rho_{\text {ex }, 0}\left\{\left[1-\frac{1}{S+1} B_{S}\left(\frac{S Q}{T}\right)-\frac{\sinh \frac{Q}{T}-\frac{Q}{T}}{\operatorname{coth} \frac{Q}{T}-1}\right]\left(1+\frac{3 J Z}{2 \varepsilon_{F}} \ln \frac{\varepsilon_{F}}{\max (Q, T)}\right)^{-2}\right. \\
\left.-3 \frac{S}{S+1} B_{S}^{2}\left(\frac{S Q}{T}\right)\right\}
\end{gathered}
$$

где Рех,0 $_{\text {. }}=\frac{3 \pi m J^{2} S(S+1) C}{2 N \varepsilon_{F} e^{2 \hbar}}$ (обычные единицы), $\quad-$ атомная концентрация.

Асимптотические значения равны:

$$
\begin{gathered}
\rho_{e x}=P_{e x, 0}\left\{\left[1-\left(\frac{Q}{3 T}\right)^{2}\right]\left(1+\frac{3 J Z}{2 \varepsilon_{F}} \ln \frac{\varepsilon_{F}}{T}\right)^{-2}-\frac{S(S+1) Q^{2}}{3 T^{2}}\right\}, Q \ll T \\
\rho_{e x}=\rho_{e x, 0}\left\{\frac{S}{S+1}\left(1+\frac{3 J Z}{2 \varepsilon_{F}} \ln \frac{\varepsilon_{F}}{O}\right)^{-2}-\frac{3 S}{S+1}\right. \\
\left.+\frac{1}{S+1}\left[\left(\frac{1}{S}-2+\frac{2 Q}{T}\right)\left(1+\frac{3 J Z}{2 \varepsilon_{F}} \ln \frac{\varepsilon_{F}}{Q}\right)^{-2}+6\right] e^{-Q / T}\right\}, e-Q / T<1
\end{gathered}
$$

Таким образом упорядочение спинов прекращает логарифомический рост сопротивления и, более того, приводит к уменьшению сопротивления благодаря замене $S(S+1)$ при логарифомическом множителе на $S^{2}$ и появлению дополнительного отрицательного слагаемого, происходящего в основном от интерференции с обычным рассеянием. Конечно, существует еще эффект возрастания сопротивления при наличии маГнитного поля, связанный с обычным искривлением әлектронной траектории. 0 нем речь пойдет несколько позже.

теперь рассмотрим как должна себя вести кривая $\rho(T)$ в различных 


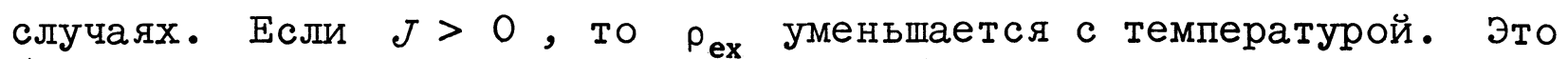
будет продолжаться до тех пор, пока $T$ не станет порядка $T_{0}$ - температуры, при которой упорядочиваются спины. Согласно предыдущему это происходит при $T \sim Q S$, т.е. при $T \sim \max \left(T_{c}, g \mu_{0} S H\right)$. После этого $\rho \rightarrow$ const. .

Если же $J<0$, то возможны разные случаи. При $Q=0$ на полной кривой $\rho(T)$ в общем случае должен быть минимум, происходящий от сложения растущей с температурой обычной части сопротивления и падающей $р$ ех • Пусть он находится при температуре $T_{\min }$ - При более низкой температуре $T_{\max }$ имеется максимум, происходящий от полюса в (15). Если $T_{0} \ll T_{\max }$ то упорядочение на кривой $\rho(T)$ проявится следующим образом. Сохраняются как $T_{\min }$, так и $T_{\max } \cdot$ Температура $T_{\max }$ не зависит от концентрации примесей. "При $T \lesssim T_{0}$ сопротивление $\rho(T) \rightarrow$ const.

Если $T_{\max } \ll T_{0} \ll T_{\min }$, то возникает то положение, которое, повидимому, чаще всего наблюдается на опыте [2]. Сохраняется минимум $\rho(T)$. Старый максимум исчезает, но появляется новый. При приближении сверху $\kappa$ To рост кривой $\rho(T)$ замедляется и сменяется падением, после чего $\rho(T)$ становится константой. Если магнитное поле отсутсвует или мало́, то $T_{0} \sim T_{c}$, так что температура максимума примерно пропорциональна концентрации магнитных примесей. Если же $g_{\mu_{0} H S} \gg T_{c}$, то температура примерно пропорциональна $H$ и не зависит от концентрации. Наконец в случае $T_{0} \gg T_{\min }$ даже при $J<0$ минимум на кривой
$\rho(T)$ отсутствует.

Во всех случаях упорядочение приводит к уменьшению наряду с этим, как уже было отмечено выше, $\rho_{\text {ord }}$ может увеличиваться под влиянием внутреннего магнитного поля. Если время между столкновениями $т \ll \frac{1}{\Omega}, \Omega=\frac{e B}{m c} \quad B$ - среднее поле в образце, то поправко к сопротивлению имеет порядок $\rho_{\text {ord }}(\Omega \tau)^{2}$.

Эту величину надо сравнивать с изменением обменного сопротивления

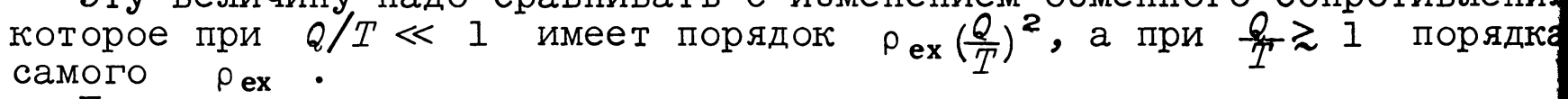

Прежде всего возникает вопрос, не может ли в отсутствии внешнего поля увеличение роrd при ферромагнитном переходе превзойти уменьшение р.е - Согласно [3] в результате ферромагнитного перехода появ ляется внутреннее поле $B \sim 4 \pi g \mu_{0} S N C$. Если определить верхний предел $4 \pi g \mu_{0} S N$ из значения индукции насыщения $B_{\text {sat }}$ для настоящих ферромагнетиков, то $P<10^{4} c \cdot$ Изменение при

Считая $\rho_{\text {ex }} / \rho_{\text {ord }} \gtrsim 10^{-2}$, получаем $\tau \ll \frac{10^{-12}}{c}$, или длину пробега $z \ll 10^{-4} / c$ : Если оценивать I по формуле $c^{c} \sim 1 / N i \sigma \sim \mathrm{I} / \mathrm{N \sigma c}$ и считать $N \sim 10^{22} \mathrm{~cm}^{-3}, \sigma \sim 10^{-16 \mathrm{~cm}^{-2}}$, то $\tau \sim 10^{-6} / \mathrm{c}, \mathrm{T.е.} \mathrm{наше}$ требование заведомо выполнено даже в том случае, если в металле нет посторонних немагнитных примесей.

Теперь рассмотрим влияние внешнего магнитного поля. В соответствии с приведенными оценками магнитное поле вызовет понижение полного сопротивления, если 


$$
\max \left(T, \mu_{0} H\right) \ll \frac{\hbar}{T} \sqrt{\frac{P_{\text {ex }}}{P_{\text {ord }}}}
$$

При $\tau \sim 10^{-3}$ справа стоит примерно $0,1^{\circ} \mathrm{K}$. Остюда следует, что полное сопротивление может как увеличиваться при включении поля, так и уменьшаться. Можно подавить возрастание обычного сопротивления добавлением немагнитных примесей. однако во всех случаях возрастание обычного сопротивления с полем есть эффект, не зависящий от температуры, а изменение обменной части, наоборот, сильно зависит от температуры.

\section{JИTEPATУPA}

1. A.A, Абрикосов, Физика (Physics), в печати.

2. A.N. Gerritsen, J.0. Linde, Physica 17, 573, 584 (1951);

18, 877 (1952); A.N. Gerritsen, Physica, 19, 61 (1953), Н.Е. Алексеевский и Ю.П. Гайдуков, ЖЮТФ, 31,947 (1956).

3. А.А. Абрикосов, Л.П. Горьков, ЖЭТФ 43, 2230 (1962). 